\title{
Application of a Damage Model to an Aluminum Alloy
}

\author{
Sylvie Castagne,* Anne Marie Habraken and Serge Cescotto \\ Department of Mechanics of Structures and Materials \\ Université de Liège \\ Chemin des Chevreuils, 1 \\ B-4000 Liège, Belgium
}

\begin{abstract}
An energy-based isotropic elastoplastic model coupled to damage is implemented in the finite element code LAGAMINE developed for more than fifteen years in the MSM department. In this model, based on the local approach of ductile fracture, effective stresses associated to damage variables are introduced. The damage law allows a continuous description of crack appearance. After a brief description of the model, its identification and its validation for an aluminum alloy are presented. Finally, the research of a global rupture criterion associated to this model is introduced.
\end{abstract}

KEY WORDS: continuum damage mechanics, finite element method, local approach, ductile fracture, triaxiality, numerical examples, experimental results.

\section{INTRODUCTION}

I

N MANY METAL forming processes such as forging, extrusion, rolling, deep drawing or cutting, the material undergoes large irreversible deformations, which could lead to the onset of cracks. To improve these industrial processes and limit the appearance of defects, engineers often use numerical simulations. These can help them in a better understanding of the process and allow parametrical studies. The prediction of fracture in structure loading processes is also of great importance. This interest of the industry in numerical methods has considerably increased the amount of research in developing constitutive models and rupture criteria.

*Author to whom correspondence should be addressed. E-mail: Sylvie.Castagne@ulg.ac.be

International Journal of DAMAGE MECHANICS, Vol. 10-January 2002 
As described by Zhu et al. (1992a), there are two common ways to predict rupture in the field of continuum mechanics. The first method consists in using classical constitutive laws coupled with the computation of rupture criteria. This is easily implemented in any numerical code but not general as it is difficult to find a single rupture criterion for all types of rupture (mode I of fracture mechanics for tensile tests and modes II and III for shear tests). The second approach is a continuum damage theory producing damage coupled constitutive laws. It is more attractive as it allows prediction of different rupture types and localization of the rupture zone. If non local approaches are used, the crack growth can be followed. Nowadays, continuum theories are a field where a tremendous amount of research work is available. Gurson (1977), Tvergaard (1982), Lemaître and Chaboche (1985) and Perzyna (1986) proposed elasto-visco-plastic and elastoplastic continuum theories for isotropic damage sensitive materials. New extensions, which cover anisotropic cases, are also available (Benzerga et al., 1997; Habraken et al., 1998).

Two types of constitutive relations are found in literature to model the coupled phenomena of elasto-plasticity and material damage, either microscopic (void growth and coalescence) or macroscopic (phenomenological). The first approach, for instance Gurson's model (Gurson, 1977), requires microscopic experimental studies to define the model's parameters and a transition from non-homogeneous microscopic material to macroscopic material, which is not always obvious. The second approach, discussed here, is based on phenomenological observations and thermodynamic considerations. It is motivated by microscopic considerations but not deduced from them. This approach can be found in works of Chaboche (1978), Lemaître (1985b), Rousselier (1987), Ju (1989), Saanouni et al. (1994), Murakami et al. (1998) and Voyiadjis and Deliktas (2000), it only requires simple macroscopic experiments.

The goal of the presented research is the validation of an isotropic elastoplastic model coupled to damage, Zhu's model (Zhu et al., 1992b), and the determination of a rupture criterion coupled to this model. The used damage theory is an adaptation of the local approach of ductile fracture, which is a prediction method based on local stress and strain fields at every point of a structure. The damage law has to allow the localization of the initiation site as well as the propagation direction of the cracks. The model has been established employing the formulation of the irreversible thermodynamics and effective state variables linked to damage variables.

To check the validity of the model, we use a set of results collected from experiments on an annealed aluminum-copper alloy (2024) manufactured for aeronautic industry and available in the form of tubes (Figure 1). The first stage of the study consists in the identification of the model for this material 


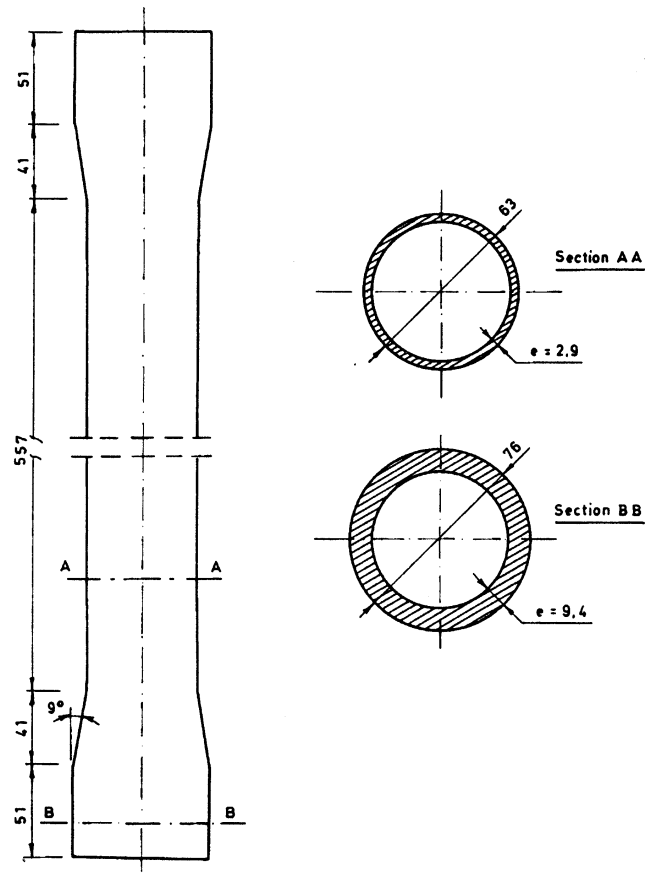

Figure 1. Description of the tube.

before beginning the validation stage by numerical simulations of several experiments chosen in such a way that a wide range of mechanical states are covered.

\section{MODEL DESCRIPTION}

The fracture prediction method is a coupled theory where damage is included in the constitutive laws. It is based on local stress, strain and damage fields at every point of the structure. We consider we have detected a crack when critical fracture conditions are achieved in a representative volume element. In this way, we can use a finite element formulation to analyze fracture processes.

\section{Damage Characterization}

Damage can be defined as a collection of permanent microstructural changes modifying thermomechanical properties (e.g., stiffness, strength, anisotropy, etc.) and brought in a material by a set of irreversible physical 
microcraking processes resulting from the application of thermomechanical loading (Taljera, 1985). For the isotropic law, two scalar damage variables are introduced in the model (Ladavéze, 1984), by which not only the elastic modulus but also the Poisson's ratio can vary with the damage growth: $d$, the deviatoric one and $\delta$, the volumetric one (see relation (1)). They represent the local material degradation, which reflects the various types of damage at the micro-scale level due to nucleation, growth and coalescence of voids, micro-cracks and other microscopic defects. Contrary to Gurson's approach, damage is not strictly connected to void volume fraction even if this is the revealing phenomenon in ductile fracture, the damage variables $d$ and $\delta$ are not quantitatively linked to void volume fraction. Experimentally, it has been shown that the ratio $\tau=\delta / d$ is close to a constant in tensile state for different materials (Gattoufi, 1984).

The additive decomposition of the total strain rate tensor $\underline{\dot{\varepsilon}}$, symmetric part of the velocity gradient, into elastic $\underline{\dot{\varepsilon}}_{e}$ and plastic $\underline{\varepsilon}_{p}$ components is used. With the hypothesis of elastic energy equivalence (Cordebois and Sidoriff, 1979; Cordebois, 1983), the damaged material state can be replaced by a fictitious undamaged material state which is characterized by effective stresses and strains (overlined variables) and through which damage variables are introduced in the model:

$$
\begin{gathered}
\underline{\bar{\sigma}}^{\prime}=\frac{\underline{\sigma^{\prime}}}{1-d} \quad \bar{\sigma}_{m}=\frac{\sigma_{m}}{1-\delta} \\
\underline{\bar{\varepsilon}}_{e}^{\prime}=(1-d) \underline{\varepsilon}_{e}^{\prime} \quad \bar{\varepsilon}_{m}=(1-\delta) \varepsilon_{m}
\end{gathered}
$$

where $\sigma_{m}$ is the mean value of the stress tensor, $\underline{\sigma}^{\prime}$ its deviator, $\underline{\varepsilon}_{e}^{\prime}$ the elastic strain tensor deviator and $\varepsilon_{m}$ the mean value of the strain tensor. Equations (1) and (2) imply the equality of the complementary elastic energy. The damage variables are given by (3):

$$
D=1-\sqrt{\frac{\bar{E}}{E_{o}}} \quad d=1-\sqrt{\frac{\bar{G}}{G_{o}}} \quad \delta=1-\sqrt{\frac{\bar{\chi}}{\chi_{o}}}
$$

where $\bar{E}$ is the Young's modulus, $\bar{G}$ the shear modulus, $\bar{\chi}$ the bulk modulus for damaged state, $E_{0}, G_{0}$ and $\chi_{0}$ the constant values for undamaged state. The model presented here uses only the two variables $d$ and $\delta ; D$ is the classical damage variable. These three variables are linked by relation (5) 
obtained substituting the relations between the elastic moduli (4) into relations (3):

$$
\begin{gathered}
G_{0}=\frac{E_{0}}{2\left(1+v_{0}\right)} \quad \chi_{0}=\frac{E_{0}}{3\left(1-2 v_{0}\right)} \\
\bar{G}=\frac{\bar{E}}{2(1+\bar{v})} \quad \bar{\chi}=\frac{\bar{E}}{3(1-2 \bar{v})} \\
\delta=1-(1-D)(1-d) \sqrt{\frac{3 G_{0}-E_{0}}{3 G_{0}(1-d)^{2}-E_{0}(1-D)^{2}}}
\end{gathered}
$$

with $v_{0}$ and $\bar{v}$ the Poisson's coefficient for undamaged and damage state, respectively. Finally, combining the previous relations, we can write the two classical elastic parameters $\bar{E}$ and $\bar{v}$ as functions of the two independent damage variables $d$ and $\delta$.

\section{Thermodynamic Approach}

Thermodynamics of irreversible phenomena gives the necessary basic principles to develop a model based on a continuous evolution of damage. The existence of the damage potential and the related damage evolution surface is postulated a priori in the framework of this approach. Murakami and Hayakawa (Hayakawa and Murakami, 1998; Murakami et al., 1998) experimentally validate the existence of the damage surface, the assumption of the associated flow rule and the normality law of damage evolution, i.e., the existence of the damage potential.

Table 1 summarizes the independent variables of the present model and their associated thermodynamic forces.

The Helmhotz specific free energy $\Psi$ is taken as thermodynamic potential:

$$
\rho \psi\left(\underline{\varepsilon}_{e}, T, d, \delta, \alpha, \beta\right)=W_{e}\left(\underline{\varepsilon}_{e}, T, d, \delta\right)+\Psi_{p}(\alpha, T)+\Psi_{d}(\beta, T)
$$

Table 1. State variables and associated thermodynamic forces.

\begin{tabular}{ll}
\hline State Variables & Associated Thermodynamic Forces \\
\hline Elastic strain $\underline{\varepsilon}_{e}$ & Cauchy stress $\underline{\sigma}$ \\
Accumulated plastic & Plastic hardening threshold $R$ \\
energy variable $\alpha$ & \\
Damage variables $d, \delta$ & Damage energy released rates $Y_{d}, Y_{\delta}$ \\
Overall damage $\beta$ & Damage strengthening threshold $B$ \\
Temperature $T$ & Entropy $S$ \\
\hline
\end{tabular}


where $\Psi_{p}$ is the free energy due to plastic hardening, $\Psi_{d}$ the free energy due to damage hardening and $W_{e}$ is the elastic strain energy, which can be divided into deviatoric strain energy $W_{e}^{\prime}$ and volumetric dilatation energy $W_{m}$ :

$$
W_{e}=W_{e}^{\prime}+W_{m}
$$

in which:

$$
\begin{gathered}
W_{e}^{\prime}=\frac{1}{2} \underline{\sigma^{\prime}}: \underline{\varepsilon}_{e}^{\prime}=G_{0}(1-d)^{2}\left(\underline{\varepsilon}_{e}^{\prime}: \underline{\varepsilon}_{e}^{\prime}\right)=\frac{\underline{\sigma}^{\prime}: \underline{\sigma}^{\prime}}{4 G_{0}(1-d)^{2}} \\
W_{m}=\frac{3}{2} \sigma_{m} \varepsilon_{m}=\frac{9 \chi_{0}(1-\delta)^{2}}{2} \varepsilon_{m}^{2}=\frac{\sigma_{m}^{2}}{2 \chi_{0}(1-\delta)^{2}}
\end{gathered}
$$

The complementary energy $\pi$ is obtained from Legendre transformation with respect to strain:

$$
\rho \pi(\underline{\sigma}, T, d, \delta, \alpha, \beta)=\underline{\sigma}: \underline{\varepsilon}_{e}-\rho \psi\left(\underline{\varepsilon}_{e}, T, d, \delta, \alpha, \beta\right)
$$

Following the standard thermodynamic procedure, the associated thermodynamic forces are given by derivation of (6) with respect to the state variables:

$$
\begin{aligned}
\underline{\sigma^{\prime}} & =\frac{\rho \partial \psi}{\partial \underline{\varepsilon}_{e}^{\prime}}=2 G_{0}(1-d)^{2} \underline{\varepsilon}_{e}^{\prime} \quad \sigma_{m}=\frac{\rho \partial \psi}{\partial \varepsilon_{m}}=3 \chi_{0}(1-\delta)^{2} \varepsilon_{m} \\
R & =\frac{\rho \partial \psi}{\partial \alpha}=\frac{\partial \Psi_{p}(\alpha, T)}{\partial \alpha} \quad B=\frac{\rho \partial \psi}{\partial \beta}=\frac{\partial \Psi_{d}(\beta, T)}{\partial \beta} \\
Y_{d} & =\frac{\rho \partial \psi}{\partial d}=-\frac{\underline{\sigma}^{\prime}: \underline{\sigma^{\prime}}}{2 G_{0}(1-d)^{3}}=-\frac{2 W_{e}^{\prime}}{1-d} \quad Y_{\delta}=\frac{\rho \partial \psi}{\partial \delta}=-\frac{\sigma_{m}^{2}}{\chi_{0}(1-\delta)^{3}}=-\frac{2 W_{m}}{1-\delta}
\end{aligned}
$$

In the present model, the Clausius-Duhem inequality can be written:

$$
\underline{\sigma}: \underline{\dot{\varepsilon}}_{p}-R \dot{\alpha}-Y_{d} \dot{d}-Y_{\delta} \dot{\delta}-B \dot{\beta}-\frac{1}{T} \underline{\operatorname{grad}} T \cdot \underline{q} \geq 0
$$

where $q$ is the heat flux. Within the assumption of uncoupling between mechanical and thermal dissipations and of the independence of energy 
dissipation between plastic flow and damage processes, (12) can be separated into three parts:

$$
\begin{gathered}
\underline{\sigma}: \underline{\dot{\varepsilon}}_{p}-R \dot{\alpha} \geq 0 \\
-Y_{d} \dot{d}-Y_{\delta} \dot{\delta}-B \dot{\beta} \geq 0 \\
-\frac{1}{T} \underline{\operatorname{grad}} T \cdot \underline{q} \geq 0
\end{gathered}
$$

This suggests that there exists a plastic dissipative potential $F_{p}$ and a damage dissipative potential $F_{d}$, which are independent. Such a choice is justified by the physics: the dissipation associated with the plastic deformation in polycrystalline materials is mainly produced by dislocation motion under the applied stress while the damage dissipation is governed by the release of internal energy due to the development of microscopic cavities (Hayakawa and Murakami, 1998). Introducing the Lagrange multipliers $\dot{\lambda}_{p}$ and $\dot{\lambda}_{\delta}$, the dissipation power $\Phi$ can be written:

$$
\Phi=\underline{\sigma}: \underline{\dot{\varepsilon}}_{p}-R \dot{\alpha}-Y_{d} \dot{d}-Y_{\delta} \dot{\delta}-B \dot{\beta}-\frac{1}{T} \underline{\operatorname{grad}} T \cdot \underline{q}-\dot{\lambda}_{p} F_{p}-\dot{\lambda}_{\delta} F_{\delta}
$$

Now, the thermodynamic problem reduces in finding the extremum of the function $\Phi$ :

$$
\begin{gathered}
\frac{\partial \Phi}{\partial \underline{\sigma}}=0 \Rightarrow \dot{\varepsilon}_{p}=\dot{\lambda}_{p} \frac{\partial F_{p}}{\partial \underline{\sigma}} \\
\frac{\partial \Phi}{\partial R}=0 \Rightarrow \dot{\alpha}=-\dot{\lambda}_{p} \frac{\partial F_{p}}{\partial R} \\
\frac{\partial \Phi}{\partial Y_{d}}=0 \Rightarrow \dot{d}=-\dot{\lambda}_{d} \frac{\partial F_{d}}{\partial Y_{d}} \\
\frac{\partial \Phi}{\partial Y_{\delta}}=0 \Rightarrow \dot{\delta}=-\dot{\lambda}_{d} \frac{\partial F_{p}}{\partial Y_{\delta}} \\
\frac{\partial \Phi}{\partial B}=0 \Rightarrow \dot{\beta}=-\dot{\lambda}_{d} \frac{\partial F_{d}}{\partial B}
\end{gathered}
$$

\section{Plasticity and Damage Evolution}

Damage evolution is represented in this model by a decrease of the elastic modulus as proposed by Lemaitre (1985b), which is different from a porosity concept. According to effective stress and strain definition in a 
fictitious undamaged material (see relation (1)), we can apply a classical plasticity model where no volumetric inelastic strain appears even when damage progresses. The plastic yield surface (18) is obtained using a vonMises plasticity criterion based on energy:

$$
F_{p}=\sqrt{W_{e}^{\prime}}-R_{0}-R(\alpha)=\sqrt{\frac{\underline{\sigma^{\prime}}: \underline{\sigma^{\prime}}}{4 G_{0}(1-d)^{2}}}-R_{0}-R(\alpha)=\frac{\bar{\sigma}_{e q}}{\sqrt{6 G_{0}}}-R_{0}-R(\alpha)
$$

where $\left(R+R_{0}\right)$ is the yield stress, $R$ the plastic hardening term depending on the internal variable linked to plasticity $\alpha, R_{0}$ the initial yield stress. As explained further, volumetric strain induces damage evolution which modifies the effective stress.

In the current literature, three kinds of damage evolution criteria are adopted: strain-based criteria (Benallal et al., 1988; Lemaître, 1985a), stress-based criteria (Chow and Wang, 1987, 1988) and energy-based criteria (Cordebois, 1983; Ladevéze, 1984), which are the most natural ones. Ladevèze proposes the energy-based expression (19) for the case with two damage variables (Ladevéze, 1984). The damage evolution surface where deviatoric and volumetric deformation energies appear is thus given by:

$$
F_{d}=-Y_{d}-\langle\tau\rangle Y_{\delta}-B_{0}-B(\beta)=\frac{2 W_{e}^{\prime}}{1-d}+\langle\tau\rangle \frac{2 W_{m}}{1-\delta}-B_{0}-B(\beta)
$$

where $B$ is the damage strengthening depending on the internal variable $\beta$ linked to the micro-voids state and added to the initial threshold value $B_{0}$, $W_{e}^{\prime}$ and $W_{m}$ are the deviatoric and volumetric energies, respectively.

The approach proposed here uses the effective stress concept. Hence, there is no direct use of the hydrostatic stress in the plastic potential like in Gurson's type approach, as it does not appear in the undamaged expression of the plastic potential. Nevertheless, the effect of the hydrostatic stress is taken into account in the damage potential. So, in the coupled approach, where we impose that the stresses and internal variables are returned back to plastic and damage surface simultaneously, the volume variation due to void growth has an influence on both plastic and damage behavior.

$\langle\tau\rangle$ is defined by $\delta / d$ in tensile state and 0 in compression state since the volumetric damage parameter $\delta$ is not affected by compression. This means, $\delta$ cannot decrease in compression state, it just does not increase 
anymore. In tensile state, $\langle\tau\rangle$ is considered as a constant in the model implemented here.

According to relations (17) the plastic (20) and the damage (21) evolution behaviors are given by a normality law:

$$
\begin{gathered}
\dot{\varepsilon}_{p}=\dot{\lambda}_{p} \frac{\partial F_{p}}{\partial \underline{\sigma}}=\frac{\dot{\lambda}_{p} \underline{\sigma}^{\prime}}{2(1-d) \sqrt{G_{0}\left(\underline{\sigma^{\prime}}: \underline{\sigma^{\prime}}\right)}} \\
\dot{\alpha}=-\dot{\lambda}_{p} \frac{\partial F_{p}}{\partial R}=\dot{\lambda}_{p} \\
\dot{\delta}=-\dot{\lambda}_{d} \frac{\partial F_{d}}{\partial Y_{\delta}}=\langle\tau\rangle \dot{\lambda}_{d} \\
\dot{d}=-\dot{\lambda}_{d} \frac{\partial F_{d}}{\partial Y_{d}}=\dot{\lambda}_{d} \\
\dot{\beta}=-\dot{\lambda}_{d} \frac{\partial F_{d}}{\partial B}=\dot{\lambda}_{d}
\end{gathered}
$$

The volumetric damage $\delta$ increases in tensile state but not in compression state.

The isotropic (22) plastic hardening rule is expressed as follows:

$$
\dot{R}=\frac{d R}{d \alpha} \dot{\alpha}=\frac{d R}{d \alpha} \dot{\lambda}_{p}
$$

The kinematic hardening model, used for cyclic loading in general, is implemented in the original version of the constitutive law. For clarity's sake, we do not present the equations relative to this part of the model, as they are not exploited here.

Relation (20) provides the link between $\dot{\alpha}$ and the rate of equivalent plastic strain by relation (23). This is a direct consequence from the choice of an energy-based von-Mises criterion (18) instead of the classical form of von-Mises yield criterion.

$$
\dot{\alpha}=\sqrt{6 G}(1-d) \dot{\varepsilon}_{e q}^{p} \text { with } \dot{\varepsilon}_{e q}^{p}=\left(\frac{2}{3} \dot{\varepsilon}_{i j}^{p} \dot{\varepsilon}_{i j}^{p}\right)^{1 / 2}
$$

Thanks to elastic energy equivalence choice, the formulation of the yield function in damage state is equivalent to that in virgin state. So, we can evaluate the plastic deformation rate in virgin state by the plastic flow rule:

$$
\overline{\dot{\varepsilon}}_{p}=\dot{\lambda}_{p} \frac{\partial F_{p}}{\partial \underline{\underline{\sigma}}}=\frac{\dot{\lambda}_{p} \overline{\overline{\sigma^{\prime}}}}{2 \sqrt{G_{0}\left(\underline{\overline{\sigma^{\prime}}}: \underline{\overline{\sigma^{\prime}}}\right)}}
$$




$$
\dot{\alpha}=\dot{\lambda}_{p}=\sqrt{6 G_{0}} \bar{\varepsilon}_{e q}^{p}
$$

Thus, the relation between plastic deformation in damage and virgin state is:

$$
\overline{\dot{\varepsilon}}_{e q}^{p}=(1-d) \dot{\varepsilon}_{e q}^{p}
$$

Damage hardening rule is given by:

$$
\dot{B}=\frac{d B}{d \beta} \dot{\beta}=\frac{d B}{d \beta} \dot{\lambda}_{d}
$$

Finally, plastic (28) and damage (29) loading/unloading rules are expressed in the Kuhn-Tucker form by:

$$
\begin{aligned}
& F_{p} \leq 0, \quad \dot{\lambda}_{p} \geq 0, \quad \dot{\lambda}_{p} F_{p}=0 \\
& F_{d} \leq 0, \quad \dot{\lambda}_{d} \geq 0, \quad \dot{\lambda}_{d} F_{d}=0
\end{aligned}
$$

If $F_{p}<0$ then $\dot{\lambda}_{p}=0$ and the process is elastic-damage. For loading, $\dot{\lambda}_{p}>0$ and $F_{p}=0$. If $F_{d}<0$, the damage criterion is not satisfied and $\dot{\lambda}_{d}=0$ implies $\dot{d}=\dot{\delta}=0$ and no further damage takes place. If $\dot{\lambda}_{d}>0$ further damage is taking place and $F_{d}=0$. Therefore, the damage evolution surface separates the damage domain from the undamaged domain.

The Lagrange multipliers are determined by requiring plastic consistency condition $\dot{F}_{p}=0$ and damage consistency condition $\dot{F}_{d}=0$, simultaneously. A rather lengthy derivation gives:

$$
\begin{aligned}
& \dot{\lambda}_{p}=\frac{A_{1}}{C}\left(\underline{\dot{\varepsilon}^{\prime}}: \underline{\sigma^{\prime}}\right)+\frac{B_{1}}{C}\left(\dot{\varepsilon}_{m} \sigma_{m}\right) \\
& \dot{\lambda}_{d}=\frac{A_{2}}{C}\left(\underline{\dot{\varepsilon}^{\prime}}: \underline{\sigma^{\prime}}\right)+\frac{B_{2}}{C}\left(\dot{\varepsilon}_{m} \sigma_{m}\right) \\
& \underline{\dot{\sigma}^{\prime}}=2 G(1-d)^{2} \underline{\dot{\varepsilon}^{\prime}}+\left[\frac{A_{3}}{C}\left(\underline{\dot{\varepsilon}^{\prime}}: \underline{\sigma^{\prime}}\right)+\frac{B_{3}}{C}\left(\dot{\varepsilon}_{m} \sigma_{m}\right)\right] \underline{\sigma^{\prime}}+\underline{\Omega \sigma^{\prime}}-\underline{\sigma^{\prime} \Omega} \\
& \dot{\sigma}_{m}=3 \chi(1-\delta)^{2} \dot{\varepsilon}_{m}+\left[\frac{A_{4}}{C}\left(\underline{\dot{\varepsilon}^{\prime}}: \underline{\sigma^{\prime}}\right)+\frac{B_{4}}{C}\left(\dot{\varepsilon}_{m} \sigma_{m}\right)\right] \sigma_{m}
\end{aligned}
$$


where, as we work in a large strain assumption the last two terms of the third relation are the contribution of Jaumann stress rate and $\underline{\Omega}$ is the material spin vector. The coefficients $A_{i}, B_{i}$ and $C$ are given by:

$$
\begin{aligned}
C & =(2+\langle\eta\rangle)\left[1+\frac{2\langle\tau\rangle^{2}\langle\xi\rangle W_{m}}{(1-\delta)^{2}}\right]+\frac{2\langle\xi\rangle W_{e}^{\prime}(2-\langle\eta\rangle)}{(1-d)^{2}} \\
A_{1} & =\langle\eta\rangle\left[1+\frac{2\langle\tau\rangle^{2}\langle\xi\rangle W_{m}}{(1-\delta)^{2}}-\frac{2\langle\xi\rangle W_{e}^{\prime}}{(1-d)^{2}}\right] / \sqrt{W_{e}^{\prime}} \\
B_{1} & =-\frac{12\langle\tau\rangle\langle\eta\rangle\langle\xi\rangle \sqrt{W_{e}^{\prime}}}{(1-d)(1-\delta)} \\
A_{2} & =\frac{4\langle\xi\rangle}{1-d} \quad B_{2}=\frac{6\langle\tau\rangle\langle\xi\rangle(2+\langle\eta\rangle)}{1-\delta} \\
A_{3} & =-\left[\langle\eta\rangle\left(1+\frac{2\langle\tau\rangle^{2}\langle\xi\rangle W_{m}}{(1-\delta)^{2}}-\frac{2\langle\xi\rangle W_{e}^{\prime}}{(1-d)^{2}}\right)+\frac{16\langle\xi\rangle W_{e}^{\prime}}{(1-d)^{2}}\right] /\left(2 W_{e}^{\prime}\right) \\
B_{3} & =-\frac{6\langle\tau\rangle\langle\xi\rangle(4-\langle\eta\rangle)}{(1-d)(1-\delta)} \\
A_{4} & =-\frac{8\langle\tau\rangle\langle\xi\rangle}{(1-d)(1-\delta)} \quad B_{4}=-\frac{12\langle\tau\rangle^{2}\langle\xi\rangle(2+\langle\eta\rangle)}{(1-\delta)^{2}}
\end{aligned}
$$

with

$$
\begin{aligned}
& \langle\eta\rangle=\left\{\begin{array}{cc}
0 & \text { under unloading and neutral state } \\
\frac{1}{d R / d \alpha} & \text { under loading state for plastic evolution }
\end{array}\right. \\
& \langle\xi\rangle=\left\{\begin{array}{cc}
0 & \text { under unloading and neutral state } \\
\frac{1}{d B / d \beta} & \text { under loading state for damage evolution }
\end{array}\right.
\end{aligned}
$$

The plastic and damage hardening laws are not given analytically as it can be done in other approaches (Saanouni et al., 1994). In practice, two reference curves, $R$ versus $\alpha$ and $B$ versus $\beta$, are stocked as data for the computation (see next chapter).

Comparing the second and third relations in Equation (21), we have the equality between $\dot{\beta}$ and $\dot{d}$. Hereafter, we will keep only the variable $d$ in all the equations.

\section{Numerical Implementation}

This damage elastoplastic constitutive law has been implemented in the implicit static version of the finite element code LAGAMINE. A global 
step-by-step integration of the equilibrium equations is carried out to solve the whole response of the structure. The Newton-Raphson iterative technique is used with convergence criteria on out of balance forces and displacements. More details about the techniques used to resolve nonlinear finite element problems can be found in Charlier's thesis (Charlier, 1987).

The integration of the constitutive relation is performed locally at each time step with a subincremental method to determine the stresses and other states variables distributions inside the structure. The operator splitting methodology is applied: we consider an additive decomposition of the equations into the elastic and coupled plastic-damage parts. The elastic predictor is corrected using the return mapping procedure: predictor stresses and internal variables are returned to the plastic and damage surfaces (Zhu et al., 1992b). The incremental compliance matrix is computed by a perturbation technique. In order to improve the numerical accuracy and stability, and to ensure convergence at large increments, an automatic subincrementing technique is proposed. If the convergence with few subincrements is not achieved, the integration is restarted with a new improved number of subincrements.

\section{MODEL IDENTIFICATION}

Identification consists in finding model parameters with the help of experiments, theory analysis, curve fitting and finite element simulations. As most of the material data are experimentally defined, only simulations reduced to one finite element for homogeneous stress-strain states are necessary for the identification step. Such simulations allow verifying the adequacy between experimental and simulated stress-strain and damagestrain curves. The validation phase presented in the next section compares numerical results obtained with the model using data issued from the identification process and experimental results of experiments, which were not used for identification.

For all simulations, the following assumptions have been made: the material is isotropic and non-viscous, hardening is isotropic and identification is made at room temperature, we consider an isothermal loading. Compression tests for three directions of loading proved the isotropy of the material. They were realized on small cubes extracted from the tube wall.

The principal data to be introduced in the model are the effective equivalent stress-plastic strain curve $\left(\bar{\sigma}_{e q}-\bar{\varepsilon}_{e q}^{p}\right)$ and the damage strengthening threshold versus deviatoric damage curve $(B-d)$ for a uniaxial test. To compute these curves, we have to know the evolution of the uniaxial stress and of the two damage variables $d$ and $\delta(3)$ versus strain. The curve $(R-\alpha)$ 
need not be given explicitly as it is computed from the curve $\left(\bar{\sigma}_{e q}-\bar{\varepsilon}_{e q}^{p}\right)$ by the pre-processor thanks to:

$$
R=\frac{\bar{\sigma}_{e q}}{\sqrt{6 G_{0}}} \text { and } \alpha=\sqrt{6 G_{0}} \bar{\varepsilon}_{e q}^{p}
$$

The plastic hardening law $(R-\alpha)$ has exactly the same form as the effective stress-plastic strain curve (Figure 4) except the scale factor $\sqrt{6 G_{0}}$ which comes from the choice of elastic energy equivalence hypothesis in the previous chapters. Within this approach, the model is able to reproduce any experimental plastic hardening.

\section{The Tensile Tests}

The Young's and shear moduli are determined from the unloading slope of cyclic loading/unloading quasi-static tensile tests on the whole tube. According to the elasticity theory, the actual Young's modulus is given by the slope of the curve $\sigma_{1}$ versus $\varepsilon_{a}$ and the shear modulus by the slope of the curve $\sigma_{1}$ versus $2\left(\varepsilon_{a}-\varepsilon_{c}\right)$ during the elastic unloading phase where $\varepsilon_{r}, \varepsilon_{a}$ and $\varepsilon_{c}$ are radial, axial and circumferential deformations, respectively and where $\sigma_{1}$ is the stress in the axial loading direction. To compute damage variables, we consider that the phenomenon appearing at the beginning of the plasticity (dotted line in Figure 2) is neglected so that these damage variables increase monotonically from 0 (Figure $7 \mathrm{a}$ ). Using extrapolated values of Young's and shear moduli $E_{0}$ and $G_{0}$ follows the classical macroscopic damage theory as described by Lemaître and Chaboche (1985). It is assumed that the evolution of the moduli at the beginning of the curve is due to plasticity entrance and not damage.

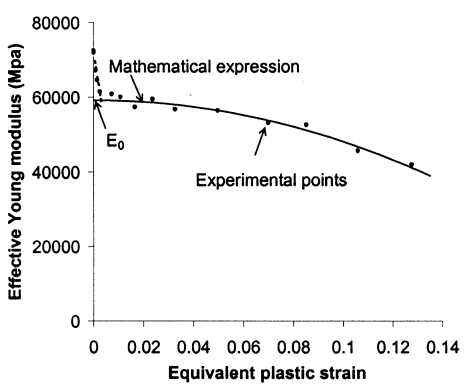

(a)

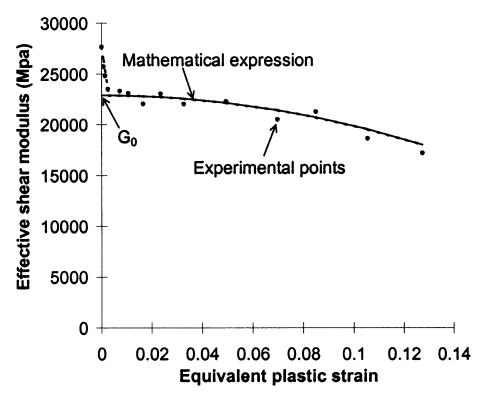

(b)

Figure 2. Tensile state. (a) effective Young's modulus; (b) effective shear modulus. 


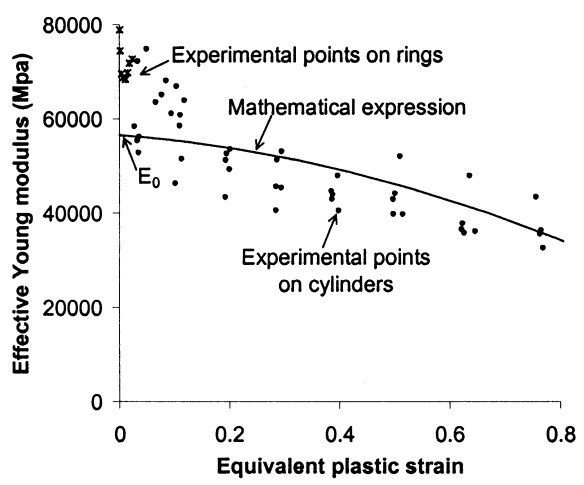

Figure 3. Effective Young's modulus (compression state).

\section{The Compression Tests}

The effective Young's modulus was measured by loading/unloading cycles on small cylinders. To improve the accuracy for the small plastic strains, cyclic compression tests were performed on a ring (slice of the tube) but those data were finally not retained for our model as the used $E_{0}$ computed from the extrapolation of the Young's modulus curve (Figure 3) seems not compatible with this new set of experiments. This difference can result from a size effect of the sample, from the different strain evaluation for small cylinders and ring slice or from the accuracy of the two different presses used to perform the experiments.

The measure of the shear modulus was not precisely realized for the compression state because of practical problems linked to the sample geometry and barreling. The determination of the damage variables thanks to Equations (3) and (4) was thus not possible. Nevertheless, the assumption of no volumetric damage in compression $(\delta=0)$ allows us to determine the deviatoric damage variable from Equation (5):

$$
d=1-(1-D) \sqrt{\frac{E_{0}}{3 G_{0}-3 G_{0}(1-D)^{2}+E_{0}(1-D)^{2}}}
$$

\section{Identification Method}

The knowledge of $(d-\varepsilon)$ curves computed as explained in the previous subsection as well as the availability of $(\sigma-\varepsilon)$ experimental curves for the tensile and compression states allow us to determine the useful data for the 
model. Indeed, as these curves depend on strain, new curves can be built making links between values corresponding to the same $\varepsilon$.

The equivalent effective stress (33), used to compute $\left(\bar{\sigma}_{e q}-\bar{\varepsilon}_{e q}^{p}\right)$, is given for a uniaxial test by:

$$
\bar{\sigma}_{e q}=\left(\frac{3}{2}{\overline{\sigma^{\prime}}}_{i j}{\overline{\sigma^{\prime}}}_{i j}\right)^{1 / 2}=\frac{\sigma_{1}}{1-d}
$$

The equivalent effective strain can be computed, as explained in (Castagne, 1998), integrating Equation (34):

$$
\overline{\bar{\varepsilon}}_{e q}^{p}=\left(1-d\left(\varepsilon_{e q}^{p}\right)\right) \dot{\varepsilon}_{e q}^{p}
$$

Finally, a unique curve is conserved taking the average between compression and tensile cases (Figure 4).

The second curve to be introduced to characterize the material is the $(B-d)$ curve. Using Equation (19) equalized to zero, we obtain relation (35) for a uniaxial test:

$$
B_{0}+B(d)=\left(\frac{1}{3 G_{0}(1-d)^{3}}+(p)\langle\tau\rangle \frac{1}{9 \chi_{0}(1-\langle\tau\rangle d)^{3}}\right) \sigma_{1}^{2}
$$

The damage threshold is given by (36):

$$
B_{0}=\left(\frac{1}{3 G_{0}}+(p)\langle\tau\rangle \frac{1}{9 \chi_{0}}\right) \sigma_{d}^{2}
$$

with $\sigma_{d}$, the initial yield stress according to the hypothesis of simultaneous plasticity and damage entrances $\left(\sigma_{d}=75 \mathrm{MPa}\right)$. A weight factor $p$ has been introduced in the model (see details in next chapter). Its value has already

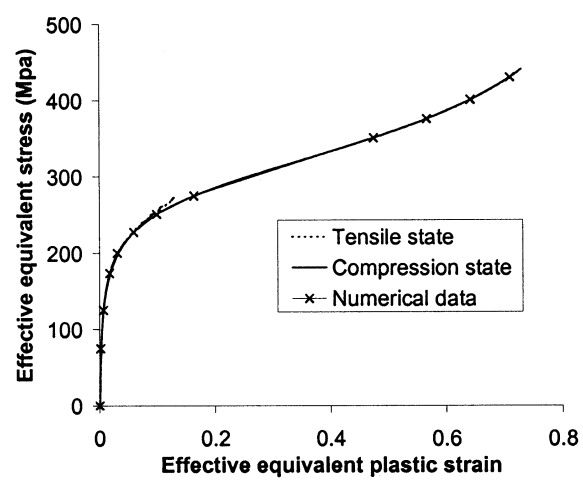

Figure 4. Effective equivalent stress vs. effective equivalent plastic strain (numerical data $=$ multilinear description). 
been taken into account in the identification phase as it modifies the $(B-d)$ curve in tensile state.

Here two different curves will be computed, one for the compression test and another one for the tensile test. To model fracture in tensile state, we introduce a limit value $d_{\text {coales, }}$, which indicates voids coalescence. As soon as this value is achieved, the slope of the $(B-d)$ curve is multiplied by the factor MP that increases the damage growth (Figure 5) and consequently the stress reduction. Without this modification, the curve is assumed to continue with the slope computed between the last two given points. In compression state, no modification is introduced as no coalescence appears.

The damage ratio $\tau$, also called tensile effect, is supposed to be a constant for the model. Actually, it varies between 1.37 and 2.30 (Figure 6). The constant value used in the simulations is 1.57 .

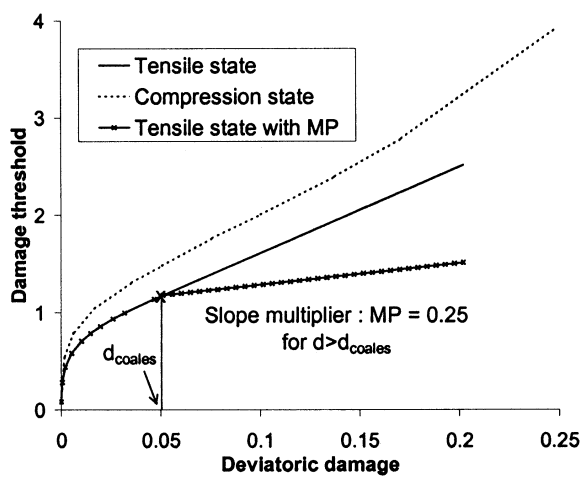

Figure 5. $(B-d)$ curve relative to tensile and compression states.

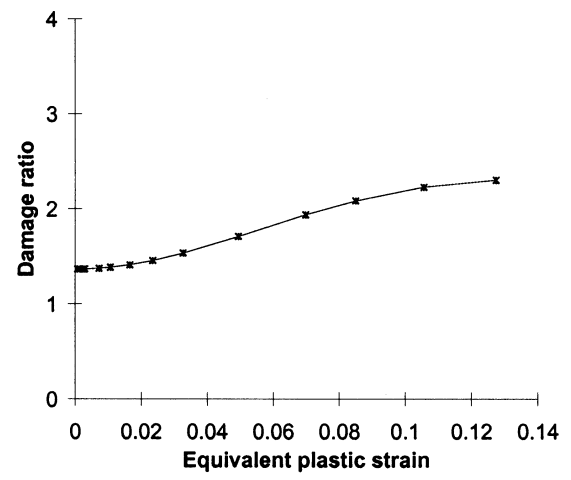

Figure 6. Tensile state: $\tau$ vs. equivalent plastic strain. 


\section{Material Data}

Curves to be introduced in the data file were plotted in the previous subsection. Figure 4 shows the numerical data corresponding to both tensile and compression states. The two upper curves of Figure 5, one for the compression state and the other one for the tensile state, are linearized to be written in the data file. Table 2 contains the numerical values of the points introduced in this file. The slope multiplier and the coalescence level will also be taken into account in the model. They are given by two scalar variables, which are not easily fitted as explained in the two following sections. They will be corrected during the validation step to obtain a better visualization of the rupture event.

Table 3 summarizes the final set of scalar data for the analyzed aluminum. We remark that the actual Young's modulus of the material is not used in

Table 2. Data curves.

\begin{tabular}{|c|c|c|c|c|c|}
\hline \multicolumn{2}{|c|}{$\left(\bar{\sigma}_{\mathbf{e q}}-\bar{\varepsilon}_{\mathbf{e w}}\right)$} & \multicolumn{2}{|c|}{$(B-d)$ Traction } & \multicolumn{2}{|c|}{$(B-d)$ Compression } \\
\hline $\bar{\varepsilon}_{\text {eq }}$ & $\bar{\sigma}_{\text {eq }}(\mathrm{MPa})$ & $D$ & $B(\mathrm{MPa})$ & $D$ & $B(\mathrm{MPa})$ \\
\hline $0.130 \times 10^{-3}$ & 75 & 0 & $0.849 \times 10^{-1}$ & 0 & $0.849 \times 10^{-1}$ \\
\hline $0.616 \times 10^{-2}$ & 125.22 & $0.512 \times 10^{-3}$ & 0.282 & $0.165 \times 10^{-2}$ & 0.547 \\
\hline $0.102 \times 10^{-1}$ & 150.14 & $0.203 \times 10^{-2}$ & 0.440 & $0.640 \times 10^{-2}$ & 0.800 \\
\hline $0.170 \times 10^{-1}$ & 173.58 & $0.523 \times 10^{-2}$ & 0.584 & $0.168 \times 10^{-1}$ & 1.044 \\
\hline $0.304 \times 10^{-1}$ & 200.06 & $0.102 \times 10^{-1}$ & 0.708 & $0.353 \times 10^{-1}$ & 1.308 \\
\hline $0.588 \times 10^{-1}$ & 227.34 & $0.147 \times 10^{-1}$ & 0.787 & $0.507 \times 10^{-1}$ & 1.481 \\
\hline $0.990 \times 10^{-1}$ & 250.94 & $0.196 \times 10^{-1}$ & 0.859 & $0.759 \times 10^{-1}$ & 1.765 \\
\hline 0.163 & 275.00 & $0.260 \times 10^{-1}$ & 0.935 & 0.100 & 2.006 \\
\hline 0.566 & 375.51 & $0.318 \times 10^{-1}$ & 0.998 & 0.136 & 2.395 \\
\hline 0.642 & 400.60 & $0.466 \times 10^{-1}$ & 1.138 & 0.171 & 2.784 \\
\hline 0.709 & 430.13 & $0.512 \times 10^{-1}$ & 1.179 & 0.248 & 3.916 \\
\hline
\end{tabular}

Table 3. Material data.

\begin{tabular}{llc}
\hline Symbol & \multicolumn{1}{c}{ Description } & Value \\
\hline$E$ & Material Young's modulus (not used) & $72,505 \mathrm{MPa}$ \\
$E_{0}$ & Initial Young's modulus & $57,852 \mathrm{MPa}$ \\
$v$ & Poisson ratio & 0.31 \\
$\tau$ & Damage ratio & 1.57 \\
$d_{\text {coales }}$ & Coalescence limit in tensile state & 0.05 \\
$\mathrm{MP}$ & Slope multiplier of $B(d)$ in tensile state & 0.25 \\
$P$ & Weight factor & 0.1 \\
\hline
\end{tabular}


the simulations. Indeed, all the equations of the model are written using the initial Young's modulus $E_{0}$ introduced previously (Figure 2).

\section{Results of the Identification}

After the model identification, deviatoric damage variable and equivalent stress represented in Figure 7 for a simulation on one finite element are in correlation with experimental results.

The rough slope modification for the damage variable in the tensile state takes account of coalescence. In fact, our experiments are not accurate enough to precisely analyze damage increase during coalescence events. Up to now, we study only a single finite element and the damage evolution after the point of coalescence aims to reproduce drastic decrease of stress (see Figure 7b) in order to model the presence of rupture and the loss of rigidity of elements representing the material points in the damaged zone. On a global mesh, when the coalescence damage threshold is achieved, the element is supposed to be as if it was taken away off the mesh. In fact, the element is still present but its rigidity is very little, which should induce a decrease of the global force curve progressively when more and more elements are affected.

Theoretically, the final slope of curves describing macroscopic fracture experiments should help to define the end of damage evolution defined by the MP factor (Figure 5). However, when we try to enhance this effect, numerical convergence problems appear, as we do not use a nonlocal formulation.

Compression state also shows a stress decrease near the rupture state.

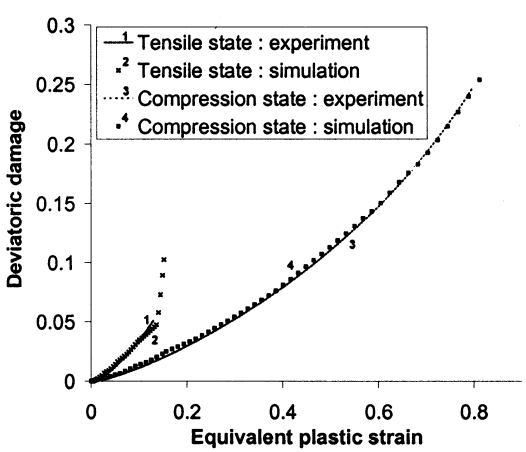

(a)

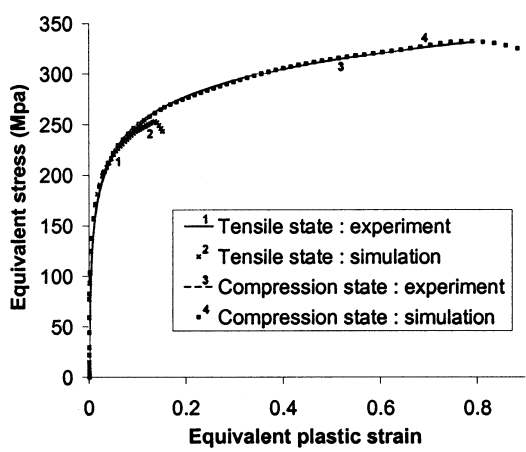

(b)

Figure 7. (a) Deviatoric damage variable; (b) equivalent stress. 


\section{VALIDATION}

Knowing the parameters to be used, three different experiments, independent of the previous ones, are modeled. They aim to reproduce a wide range of mechanical states. The finite elements used to model all the proposed experiments are 4-nodes mixed elements for the 2D state (8-nodes for 3D) with one integration point (Zhu and Cescotto, 1995).

\section{The Notch Tests}

Let us consider tensile tests realized on notched cylindrical bars (Figure 8). The relative displacement is measured on a $25 \mathrm{~mm}$ basis. Three different meshes are tested in order to analyze the mesh sensitivity of the results. The experiment has been reproduced several times with very close results, which explains why only one experimental curve is presented in Figure 9(a).

In the first simulations, very local strong damage increases with loss of convergence happened. A kinematic scalar indicator based on an idea

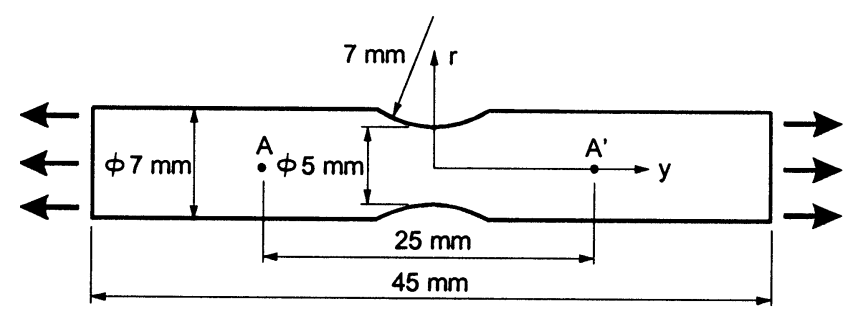

Figure 8. Notch test: sample description.

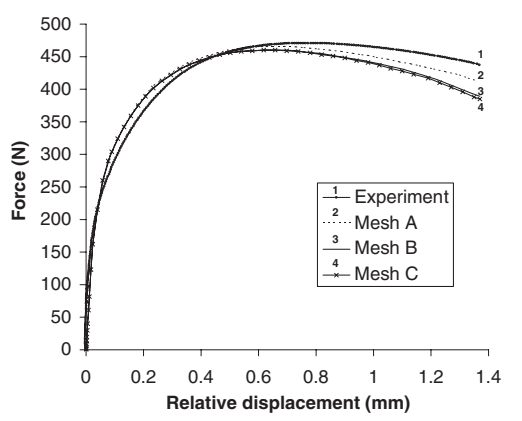

(a)

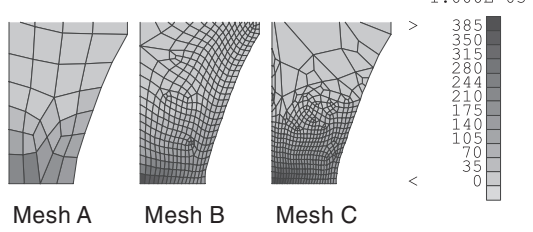

(b)

Figure 9. Model without $d_{\text {coales }}$ neither MP; (a) force-displacement; (b) deviatoric damage for a $1.35 \mathrm{~mm}$ relative displacement. 
proposed by Vilotte and used in (Pierry, 1997) proved the appearance of bifurcations. These localization phenomena were pointed out long before the rupture event. We used a viscous regularization method to improve the numerical stability and we suppressed the slope modification (MP) taking voids coalescence into account but this did not solve the problem.

An analysis of the damage map shows that this one increases more in zones where triaxiality (ratio between the mean stress and the equivalent stress) is very high. Compared to the experiment, its evolution is too important as it predicts high local damage value long before rupture. This effect is enhanced when the slope of the $(B-d)$ curve is reduced. To limit the damage growth and allow a better convergence of the simulation, we introduce in the initial model a weight factor $p$ limiting the influence of the hydrostatic energy term $W_{m}$, which involves triaxiality effects (37). This is equivalent to a modification of the damage surface shape (19).

$$
F_{d}=\frac{2 W_{e}^{\prime}}{1-d}+p\langle\tau\rangle \frac{2 W_{m}}{1-\delta}-B_{0}-B(d)
$$

The weight factor obtained is $p=0.1$. It gives a correct evolution of damage and keeps the difference of behavior between tensile and compression states. Graphs of Figure 7 were in fact drawn taking into account this factor. Indeed, the identification has been done with the final model.

The curve representing the force on Figure 9(a) is lower than the one obtained by the experiment although rupture by coalescence in tensile state has been removed from the model for those cases.

As already studied by lots of authors and for instance by Needleman and Tvergaard (1984), Figure 9(b) shows that damage increases more in some elements localized at the middle of the specimen, where triaxiality is very high and can achieve 1.6 in our example. Introducing in the model a $d_{\text {coales }}$ would allow a more important increase of the damage variable, it would induce greater convergence problems in our local approach.

\section{The Tensile Test of Perforated Specimen}

The second experiment is a tensile test on a curved bar with a hole inside (Figure 10). The hole is not exactly in the center in such a way that we can predict on which side of the hole fracture will begin and focus a camera on that side. However, only a half of the bar is modeled 


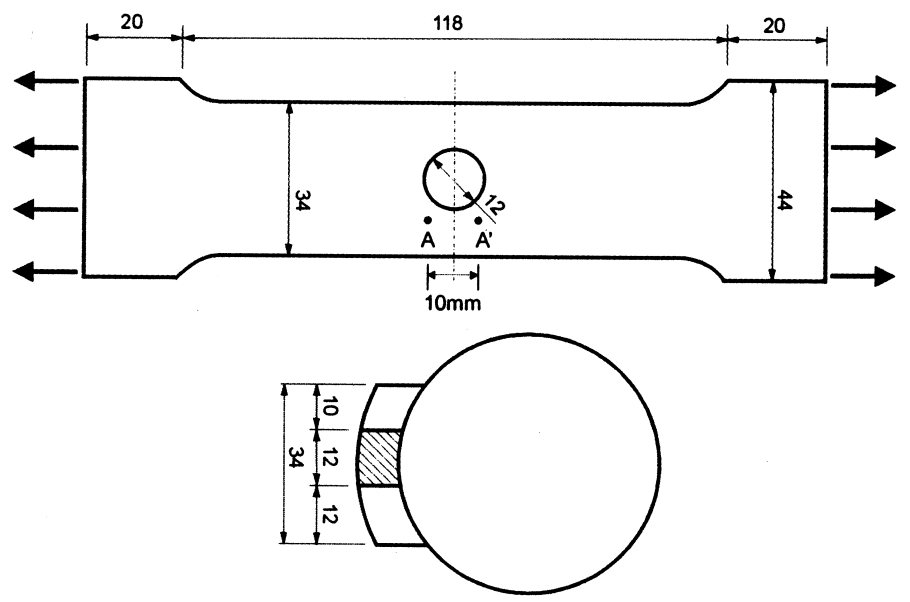

Figure 10. Tensile test of perforated specimen: geometry.

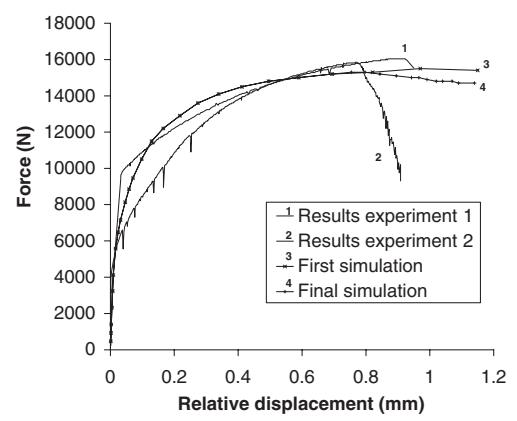

(a)

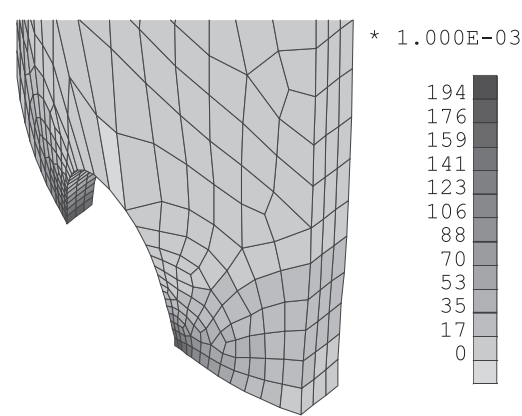

(b)

Figure 11. (a) Force vs. relative displacement; (b) final simulation: deviatoric damage for a $1.1 \mathrm{~mm}$ relative displacement.

by symmetry. The relative displacement is measured on a $10 \mathrm{~mm}$ base between two points localized on each side of the symmetry axis, in the vicinity of the hole.

The first simulation in Figure 11(a) does not include the reduction of the $(B-d)$ slope when coalescence of voids happens. Otherwise the final simulation has been realized with $d_{\text {coales }}=0.12$ and $\mathrm{MP}=0.25$. We can observe that with the second simulation, a weak decrease of the force appears at the moment corresponding to the experimental rupture. Figure 11(b) shows that the damage is maximum near the hole but its value remains low compared to the values obtained with the notch tests. Indeed, the maximum triaxiality here is 0.5 while it is 1.6 for the notch tests. 


\section{The Shear Test of Perforated Tube}

The last experiments were realized on specimens issued from the extremity of the tube and machined to obtain a zone where shear is important when the sample is compressed (Figure 12). The charge is applied using a punch which introduces shear and pressure stresses. The sample is placed on the lubricated plate of the press. Using symmetry, only one quarter of the specimen is modeled.

In Figure 13(a), the first simulation corresponds to a case with no coalescence, the second simulation has been realized with $d_{\text {coales }}=0.15$ and $\mathrm{MP}=0.5$ and the last one with the same parameters as the second simulation for the perforated specimen. For this last simulation, a reduction of the force can be observed at an imposed displacement of $7.7 \mathrm{~mm}$ followed by oscillations. Figure 13(b) shows that damage begins near the hole as checked by the experiment. Triaxiality reaches 0.6 , which implies damage
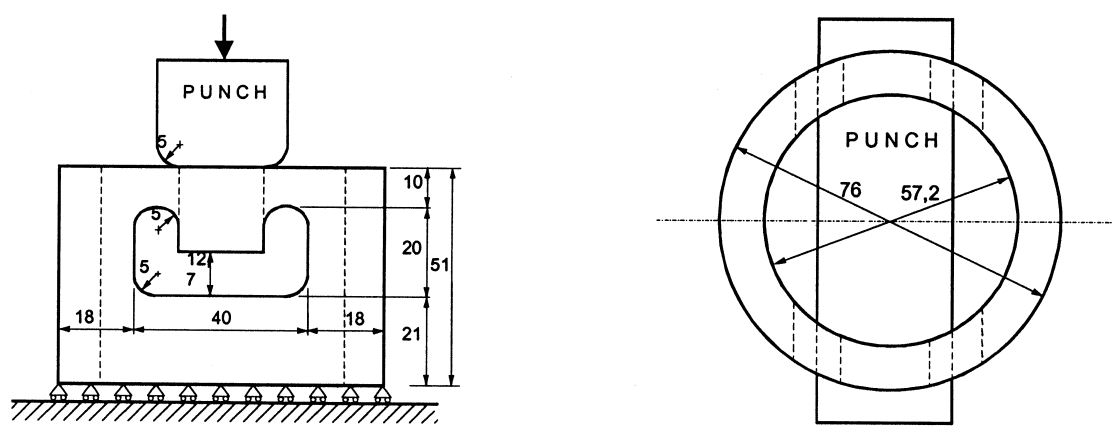

Figure 12. Shear test: geometry and loading.

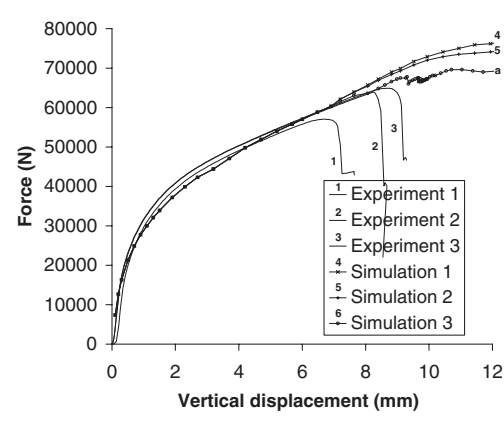

(a)

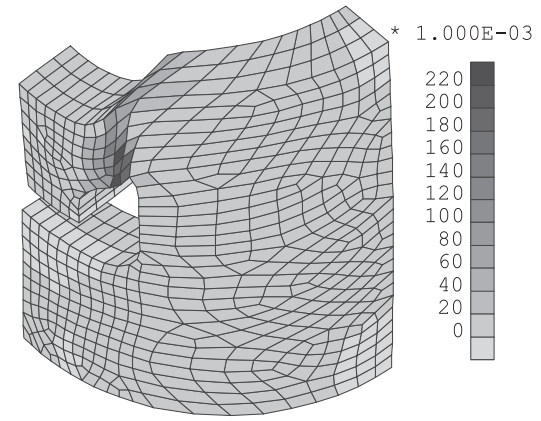

(b)

Figure 13. (a) Force vs. vertical displacement of the tool; (b) simulation 3, deviatoric displacement for a $9.1 \mathrm{~mm}$ displacement of the tool. 
values lower than in the notch tests and similar to those observed during the perforated specimen tensile test.

\section{DAMAGE AND RUPTURE CRITERION}

It can be observed that there is no volumetric inelastic strain in our model, even when damage progresses. This is in contradiction with models such as those of Gurson (1977) or Rousselier (1987) in which hypothesis relating to the growth of cavities are necessary. In our formulation, which is developed within the framework of effective stress and energy equivalence hypothesis, we use a classical energy-based plastic potential where no hydrostatic stress appears. This explains why we do not have to describe explicitly the evolution of the volumetric inelastic strain.

The preceding experiment simulations show the dependence of damage level on triaxiality. The instant of rupture does not simply correspond to the achievement of a threshold value of the damage variable but must be linked to different factors.

The initial Zhu's model already contains the effect of triaxiality on damage through the volumetric energy term. However, the notch tests clearly show that damage increase is too high to model experience reality. The modification proposed here consists in limiting the triaxiality effect in order to slow down damage increase. The coalescence damage level used in our model is a constant for the material. As it has been modified according to validation tests, it proves that it should be a function of the material stress and strain state as proposed by Thomason's criterion (Thomason, 1982) or of the triaxiality state as suggested by Brocks et al. (1995). Nevertheless, even with the present constant, the coalescence damage level is achieved earlier in a simulation in which triaxiality is high as this one increases the damage variable growth, even if the phenomenon is reduced with the modified model. The prediction of rupture from a slope variation of the global force displacement curve is not always possible; a too localized fracture cannot be detected on this curve. On the other hand, the coalescence limit $d_{\text {coales }}$ allows the detection of the micro-crack event and its following, but its constant value should become a function of material state.

Different solutions have been proposed to avoid difficulties arising from softening behaviors (Lemaitre, 2000). The convergence problems could be reduced using gradient or nonlocal methods. Contrary to the viscous regularization method, which was not sufficient here to reduce the convergence loss in our problem, these types of methods are not introduced in our model yet. Indeed, they are useful to limit convergence loss around the site of rupture initiation but they require the introduction of an internal 
length. For metals, this length scale is very weak (of the order of the distance between precipitates or inclusions). To keep a physical sense, we should use meshes thinner than those presented here before, which also means longer computational time. A first solution to improve the convergence of our model is to link the subincrement size and the damage variable. That way, we could have a more efficient integration scheme in the critical zone.

As we could not determine a constant threshold value of the damage variable $d_{\text {coales }}$ indicating that a macroscopic rupture has happened in the specimen, we have searched for a rupture criterion coupling damage and triaxiality, comparing simulated and experimental results. Nevertheless, the tests available are not numerous and accurate enough to establish this maximum value of the damage as a function of triaxiality. To get more data, we should realize new experiments on bars with various notch radiuses, which corresponds to different triaxiality values.

It is interesting to note that the difficulty in defining a threshold value on our damage variable, as it seems to depend on triaxiality, is not surprising. In fact Gurson's type models face an identical problem. The general study of Benzerga et al. (1999) presents curves of the critical porosity $f_{c r}$ depending on triaxiality $T$. According to the initial porosity value, $f_{c r}$ is quite constant or increases with low $(\approx 0.5)$ to mean values of $T(0.8-1.2)$, then decreases for mean to high $T$ values $(>1.5)$. This result from Benzerga helps to understand why, according their studied cases, authors like Needleman and Tvergaard (1984), Koplik and Needleman (1988), Brocks et al. (1995) and Brethenoux et al. (1997) have found various influences of $T$ on critical porosity. The formulation proposed in this paper is quite different from that of Benzerga. However, both represent damage process and can be compared.

\section{CONCLUSIONS}

The initial Zhu's model does not apply to the studied aluminum alloy because the damage increases too rapidly in the zones where triaxiality is high. A modification of the law that induces a less important damage increase seems to be closer from the experiment. In some cases, it allows the resolution of the convergence problems classically linked to softening models. However, the convergence is still difficult and the implementation of a length scale in the model as suggested by de Borst et al. (1999) should improve the stability and the convergence and allow our approach to really follow the crack evolution.

Cracks initiation sites are correctly predicted by this model, as well as their propagation directions. For shapes and solicitations that correspond to 
a low triaxiality, if the damage zone is not too localized, the modified model allows us to predict the sample rupture analyzing the drop in the forcedisplacement curve.

Additional experimental results are necessary for the establishment of a rupture criterion function of the material state. When known, this criterion will allow us to predict the crack evolution according to damage and triaxiality, for instance.

Finally, we should investigate the opportunity to use a nonconstant damage ratio $\tau$ instead of introducing the reduction parameter $p$ in Equation (37). Indeed, Figure 6 shows the evolution of $\tau$, which is not actually a constant for our material. Most of all, if we analyze the effect of the parameter $p$ in the second term of Equation (37), we remark that it influences the value of $\tau$ in a nonlinear way.

\section{REFERENCES}

Benallal, A., Billardon, R. and Doghri, I. (1988). Communications in Applied Numerical Methods, 4: 731-740.

Benzerga, A., Besson, J. and Pineau, A. (1997). In: Proceedings of 3éme Colloque National En Calcul Des Structures, Mai, Giens, France, pp. 20-23.

Benzerga, A., Besson, J. and Pineau, A. (1999). J. Engng. Mat. \& Tech., 121: 221-229.

Brethenoux, G., Mazataud, P., Bourgain, E., Muzzi, M. and Giusti, J. (1997). Advanced Methods in Materials Processing Defects in Studies in Applied Mechanics, Predeleanu, M. and Gilormini, P. (eds.), Elsevier, 45: 23-24.

Brocks, W., Sun, D.Z. and Hönig, A. (1995). Int. J. of Plasticity, 11: 971-989.

Castagne, S. (1998). Application d'un Modéle Isotrope Èlastoplastique Couplé à L'endommagement à un Aluminium, Student final work to get Physician Engineer Degree, Université de Liége.

Chaboche, J.L. (1978). Description Thermodynamique et Phénoménologique de la Viscoplasticité Cyclique Avec Endommagement, PhD Thesis, Université Paris 6.

Charlier, R. (1987). Approche Unifiée de Quelques Problémes non Linéaires de Mécanique des Milieux Continus par la Méthode Des Éléments Finis (Grandes Déformations Des Métaux Et Des Sols, Contact Unilatéral De Solides, Conduction Thermique et Écoulement en Milieux Poreux), PhD Thesis, Université de Liége.

Chow, C.L. and Wang, J. (1987). Engng. Frac. Mech., 27: 547-558.

Chow, C.L. and Wang, J. (1988). Engng. Frac. Mech., 30: 547-563.

Cordebois, J.P. and Sidoroff, F. (1979). Euromech, 115 Villard de Lans.

Cordebois, J.P. (1983). Critéres D'instabilité Plastique et Endommagement Ductile en Grandes Déformations. Application à L'emboutissage, $\mathrm{PhD}$ Thesis, Université Paris 6.

de Borst, R., Pamin, J. and Geers, M. (1999). Eur. J. Mech. A/ Solids., 18: 939-962.

Gattoufi, B. (1984). Effets de la Prédéformation Due au Filage Sur le Comportement Des Métaux, PhD Thesis, Université Paris.

Gurson, A.L. (1977). J. Engng. Materials Technology, 99: 2-15.

Habraken, A.M., Charles, J.F. and Cescotto, S. (1998). Damage Mech. in Engng. Materials in Studies in Applied Mechanics, Voyiadijs, G.Z., Ju, J.W. and Chaboche, J.L. (eds.), Elsevier, 46: 401-420. 
Hayakawa, K. and Murakami, S. (1998). Damage Mech. in Engng. Materials Studies in Applied Mechanics, Voyiadijs, G.Z., Ju, J.W. and Chaboche, J.L. (eds.), Elsevier, 46: 27-44.

Koplik, J. and Needleman, A. (1988). Int. J. Solids \& Structures, 24: 835-853.

Ju, J.W. (1989). Int. J. Solids \& Structures, 25: 803-833.

Ladevéze, P. (1984). Sur Une Théorie De L'endommagement, Internal Report 34, LTM, Cachan.

Lemaître, J. (1985a). J. Engng. Materials Technology, 107: 83-89.

Lemaître, J. (1985b). Comp. Meth. in Appl. Mech. Engng., 51: 31-49.

Lemaître, J. and Chaboche, J.L. (1985). Mécanique Des Matériaux Solides, Dunod, Paris.

Lemaître, J. (2000). In: Benallal, A. (ed.), Continuous Damage and Fracture, pp. 1-5, Elsevier. Murakami, S., Hayakawa, K. and Liu, Y. (1998). Int. J. of Damage Mechanics, 7: 103-128.

Needleman, A. and Tvergaard, V. (1984). J. Mech. Phys. Solids, 32: 461-490.

Perzyna, P. (1986). Int. J. Solids \& Structures, 22: 797-818.

Pierry, J. (1997). Modélisation Des Bandes De Cisaillement Et Adaptation De Maillages. Application à la Coupe Des Roches, $\mathrm{PhD}$ Thesis, Université de Liége.

Rousselier, G. (1987). Nuclear Engng. Des, 105: 97-111.

Saanouni, K., Forster, C. and Ben Hatira, F. (1994). Int. J. of Damage Mechanics, 3: 140-169.

Taljera, R. (1985). Proc. R. Soc. Lond. Ser., A399: 195-216.

Thomason, P.F. (1982). Acta Metall., 30: 279-284.

Tvergaard, V. (1982). Int. J. Solids \& Structures, 18: 659-672.

Voyiadjis, G.Z. and Deliktas, B. (2000). Comp. Meth. in Appl. Mech. Engng., 183: 159-199.

Zhu, Y.Y., Cescotto, S. and Habraken, A.M. (1992a). J. Mater. Processing Technology, 32: 197-204.

Zhu, Y.Y., Cescotto, S. and Habraken, A.M. (1992b). Computational Plasticity, Fundamentals and Applications, 6: 1455-1466.

Zhu, Y.Y. and Cescotto, S. (1995). Int. J. Num. Meth. Engng., 38: 685-716. 